\title{
Theoretical study of the Josephson current through a junction including ferromagnetic insulator
}

\author{
Masashi Yamashiro, Nobukatsu Yoshida, Yukio Tanaka and Satoshi Kashiwaya* \\ Graduate School of Science and Technology, Niigata University, Ikarashi, Niigata 950-21, \\ Japan \\ *Electrotechnical Laboratory, Tsukuba, Ibaraki 305, Japan
}

\begin{abstract}
The properties of Josephson junction including a ferromagnetic insulating layer are studied theoretically. The calculated current shows the anomalous dependence on the magnitude of the exchange interaction at the interface. The Josephson junction changes from 0junction to $\pi$-junction with the increase of the magnitude of the exchange interaction.
\end{abstract}

KEYWORDS:Josephson junction, ferromagnetic insulator, exchange interaction, $\pi$ junction

\section{INTRODUCTION}

It is well known that the properties of the Josephson junction are strongly influenced by the bound states formed around the insulator [1-5]. This bound states are current carrying states the origins of which are multiple Andreev reflection [6]. Previously, Furusaki and Tsukada derived a unified formula of the Josephson current, which is written by the coeficeints of the Andreev reflection [1,2]. This formula was extended to the Josephson junction including anisotropic superconductors [3-5]. On the other hand, Tanaka and Kashiwaya developed a theory of superconductor / ferromagnetic-insulator / superconductor (S/Fi/S) junction [7] based on the Furusaki's formula. They showed that the Josephson junction changes from 0 -junction to $\pi$-junction with the increase of the magnitude of the exchange interaction strength. In this paper, we will calculate the Josephson current in $\mathrm{S} / \mathrm{Fi} / \mathrm{S}$ junction taking into account the exchange potential as well as ordinary Hartree potential at the interface which was ignored in the previous paper [7]. As in the previous results, the Josephson current changes sign with the increase of exchange interaction strength for fixed phases difference between two superconductors. Our results inclueds several existing results as limiting cases [8-10].

\section{FORMULA AND RESULTS}

We assume the system in the clean limit with perfectly flat interface which is perpendicular to the $x$-axis and is located at $x=0$. The Fermi wave number $k_{F}$ and the effective mass $m$ are assumed to be equal both on the left (negative $x$ side) and the right (positive $x$ side) superconductors. All dynamical spin processes are neglected and the magnetic influence on the superconductivity is limited to that through the exchange field in the Fi region. Since the momentum component parallel to the interface is conserved, the Bogoliubov-de Gennes equation can be expressed as

$$
\left(\begin{array}{cc}
h_{1, \sigma}(\theta) & \Delta(x) \\
\Delta^{*}(x) & -h_{2, \sigma}(\theta)
\end{array}\right) \Psi_{\sigma}(x, \theta)=E \Psi_{\sigma}(x, \theta), \quad \sigma=\uparrow, \quad \downarrow,
$$




$$
h_{1(2), \sigma}(\theta)=-\frac{\hbar^{2}}{2 m} \frac{d^{2}}{d x^{2}}+\left(H_{i}-(+) \rho_{\sigma} H_{m}\right) \delta(x)-\frac{\hbar^{2} k_{F}^{2} \cos ^{2} \theta}{2 m}, \quad \rho_{\uparrow}=1, \quad \rho_{\downarrow}=-1 .
$$

In the above, the wave function $\Psi_{\sigma}(x, \theta)$ expresses the process where an electron-like quasiparticle is injected from the left superconductor. $H_{i}$ and $H_{m}$ represent the ordinary barrier potential and exchange interaction, respectively. The quantity $\theta$ denotes the injection angle of the quasiparticle which is measured from the normal to the interface. The energy of quasiparticles $E$ is measured from the Fermi energy $E_{F}$. We assume that the pair potential is expressed as $\Delta(x)=\Delta(T) e^{i \phi_{L}}$ for $x<0$, and $\Delta(x)=\Delta(T) e^{i \phi_{R}}$ for $x>0$. The Andreev reflection coefficient $a_{\sigma}(\phi, \theta)$ is obtained as follows.

$$
a_{\sigma}(\phi, \theta)=\frac{\Delta(T)\left[-E \sin ^{2}(\phi / 2)+i \Omega\left\{\sin (\phi / 2) \cos (\phi / 2)+\rho_{\sigma} z_{m \theta}\right\}\right]}{E^{2}\left(1+Z_{\theta}^{2}\right)-\Delta^{2}(T)\left\{\cos ^{2}(\phi / 2)+Z_{\theta}^{2}\right\}-2 i E \Omega \rho_{\sigma} z_{m \theta}} .
$$

In the Eq.(3), $\Omega=\sqrt{E^{2}-\Delta^{2}(T)}$. The phase difference between the left and right superconductor is denoted as $\phi=\phi_{L}-\phi_{R}$. Barrier parameters at the interface are given by

$$
\begin{aligned}
& z_{m \theta}=\frac{m H_{m}}{\hbar^{2} k_{F} \cos \theta}, \quad z_{i \theta}=\frac{m H_{i}}{\hbar^{2} k_{F} \cos \theta} \\
& Z_{\theta}^{2}=z_{i \theta}^{2}-z_{m \theta}^{2} .
\end{aligned}
$$

Following the formulation in Ref.[1,2], the Josephson current is obtained using the generalized coefficient of the Andreev reflection $\bar{a}_{\sigma}(\phi, \theta)$ which is obtained by analytic continuation of $E$ to $i \omega_{n}$ in $a_{\sigma}(\phi, \theta)$. The obtained result for a fixed injection angle $I(\phi, \theta)$ is expressed as

$$
I(\phi, \theta)=\frac{e \Delta(T) k_{B} T}{\hbar} \sum_{\omega_{n}} \frac{\bar{a}_{\uparrow}(\phi, \theta)+\bar{a}_{\downarrow}(\phi, \theta)-\bar{a}_{\uparrow}(-\phi, \theta)-\bar{a}_{\downarrow}(-\phi, \theta)}{2 \Omega_{n}},
$$

with $\omega_{n}=2 \pi k_{B} T(n+1 / 2)$ and $\Omega_{n}=\sqrt{\omega_{n}^{2}+\Delta^{2}(T)}$. Performing the summation of the Matsubara frequency, the whole Josephson current $J(\phi)$ is obtained as

$$
\begin{aligned}
& J(\phi)=\frac{k_{F}^{2} S}{\pi} \int_{0}^{\pi / 2} I(\phi, \theta) \cos \theta \sin \theta d \theta, \\
& I(\phi, \theta)=\frac{e \Delta^{2}(T) \sin \phi}{2 \hbar\left\{\left(1+Z_{\theta}^{2}\right)^{2}+4 z_{m \theta}^{2}\right\}}\left\{\frac{\alpha(\phi, \theta)}{\varepsilon_{A}(\phi, \theta)} \tanh \frac{\varepsilon_{A}(\phi, \theta)}{2 k_{B} T}+\frac{\beta(\phi, \theta)}{\varepsilon_{B}(\phi, \theta)} \tanh \frac{\varepsilon_{B}(\phi, \theta)}{2 k_{B} T}\right\}
\end{aligned}
$$

with following quantities

$$
\begin{aligned}
& \varepsilon_{A}^{2}(\phi, \theta)=\Delta^{2}(T) \\
& \times \frac{\left(1+Z_{\theta}^{2}\right)\left(\cos ^{2}(\phi / 2)+Z_{\theta}^{2}\right)+2 z_{m \theta}\left\{z_{m \theta}+\sqrt{z_{m \theta}^{2}+\left(\cos ^{2}(\phi / 2)+Z_{\theta}^{2}\right) \sin ^{2}(\phi / 2)}\right\}}{\left(1+Z_{\theta}^{2}\right)^{2}+4 z_{m \theta}^{2}} \\
& \varepsilon_{B}^{2}(\phi, \theta)=\Delta^{2}(T) \\
& \times \frac{\left(1+Z_{\theta}^{2}\right)\left(\cos ^{2}(\phi / 2)+Z_{\theta}^{2}\right)+2 z_{m \theta}\left\{z_{m \theta}-\sqrt{z_{m \theta}^{2}+\left(\cos ^{2}(\phi / 2)+Z_{\theta}^{2}\right) \sin ^{2}(\phi / 2)}\right\}}{\left(1+Z_{\theta}^{2}\right)^{2}+4 z_{m \theta}^{2}}
\end{aligned}
$$




$$
\begin{aligned}
& \alpha(\phi, \theta)=\frac{1}{2}\left\{\left(1+Z_{\theta}^{2}\right)-\frac{z_{m \theta}\left(\cos \phi+Z_{\theta}^{2}\right)}{\sqrt{z_{m \theta}^{2}+\left(\cos ^{2}(\phi / 2)+Z_{\theta}^{2}\right) \sin ^{2}(\phi / 2)}}\right\} \\
& \beta(\phi, \theta)=\frac{1}{2}\left\{\left(1+Z_{\theta}^{2}\right)+\frac{z_{m \theta}\left(\cos \phi+Z_{\theta}^{2}\right)}{\sqrt{z_{m \theta}^{2}+\left(\cos ^{2}(\phi / 2)+Z_{\theta}^{2}\right) \sin ^{2}(\phi / 2)}}\right\} .
\end{aligned}
$$

The quantity $S$ expresses the magnitude of the section of the junction. Figs.(四) and (22) show the calculated Josephson current $J(\phi)$ and maximum Josephson current $J_{C}$ for fixed $z_{i}=1$ and several values of $z_{m}\left(z_{i(m)}=\cos \theta z_{i(m) \theta}\right)$, which are normalized by the resistance in the normal state

$$
R_{N}^{-1}=\frac{e^{2} k_{F}^{2} S}{\pi^{2} \hbar} \int_{0}^{\pi / 2} \frac{\cos \theta \sin \theta}{1+\left(z_{i \theta}-\rho_{\sigma} z_{m \theta}\right)^{2}} d \theta
$$

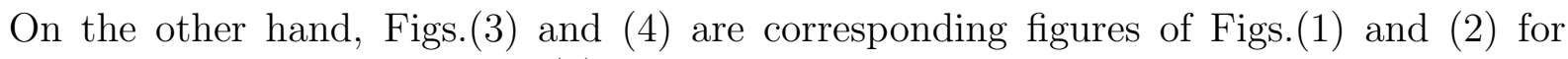
fixed $z_{m}=1$. As shown in Fig.(11), when the magnitude of $z_{m}$ becomes larger than that of $z_{i}$, the Josephson junction changes from 0 -junction to $\pi$-junction. For large $z_{m}$ or $z_{i}$, the temperature dependence of the maximum Josephson current $J_{C}$ [Figs.(2),(44)] shows similar behavior to that found in the tunneling limit of the non-magnetic barrier by Ambegaokar and Baratoff [10]. For fixed $z_{m}$, with the increase of $z_{i}$, the Josephson junction changes from $\pi$-junction to 0 -junction [Fig.(3)].

In this paper, we have developed a general formula of the d.c. Josephson current between the superconductor / ferromagnetic-insulator / superconductor junction taking into account both ordinary barrier potential as well as exchange interaction. The essence of the physics in our previous paper [7] does not change due to the existence of the ordinary potential barrier. We hope the drastical change from 0 junction to $\pi$ junction will be observed experimentally near future.

\section{REFERENCES}

1. Furusaki A and Tsukada M (1990) Physica C 165\&166: 967-968

2. Furusaki A and Tsukada M (1991) Solid State Commun. 78: 299-302

3. Tanaka Y and Kashiwaya S (1996) Phys. Rev. B 53: R11957-R11960

4. Tanaka Y and Kashiwaya S (1996) Phys. Rev. B 53: 9371-9381

5. Tanaka Y and Kashiwaya S (1997) Phys. Rev. B 56: 892-912

6. Andreev AF (1964) Sov. Phys. JETP 19: 1228-1231

7. Tanaka Y and Kashiwaya S (1997) Physica C 274: 357-363

8. Shiba H and Soda T (1969) Prog. Theor. Phys. 41: 25-44

9. Kulik IO and Omel'yanchuk (1978) Sov. J. Low Temp. Phys. 4: 142-

10. Ambegaokar V and Baratoff A (1963) Phys. Rev. Lett. 10: 486-489 


\section{FIGURE CAPTIONS}

Figure 1: Normalized $J(\phi)$ is plotted as a function of $\phi / \pi$, with a: $z_{m}=0.5, \mathrm{~b}: z_{m}=1.5$, $\mathrm{c}: z_{m}=2$ and $\mathrm{d}: z_{m}=5 . z_{i(m)}=\cos \theta z_{i(m) \theta}$

Figure 2: Normalized $J_{C}$ is plotted as a function of $T / T_{C}$, with a: $z_{m}=0.5$, b: $z_{m}=1$, c: $z_{m}=2$ and $\mathrm{d}: z_{m}=5 . \quad z_{i(m)}=\cos \theta z_{i(m) \theta}$

Figure 3: Normalized $J(\phi)$ is plotted as a function of $\phi / \pi$, with a: $z_{i}=0.5, \mathrm{~b}: z_{i}=1.5$, c: $z_{i}=2$ and $\mathrm{d}: z_{i}=5 . \quad z_{i(m)}=\cos \theta z_{i(m) \theta}$

Figure 4: Normalized $J_{C}$ is plotted as a function of $T / T_{C}$, with a: $z_{i}=0.5, \mathrm{~b}: z_{i}=1, \mathrm{c}: z_{i}=2$ and $\mathrm{d}: z_{i}=5 . \quad z_{i(m)}=\cos \theta z_{i(m) \theta}$ 


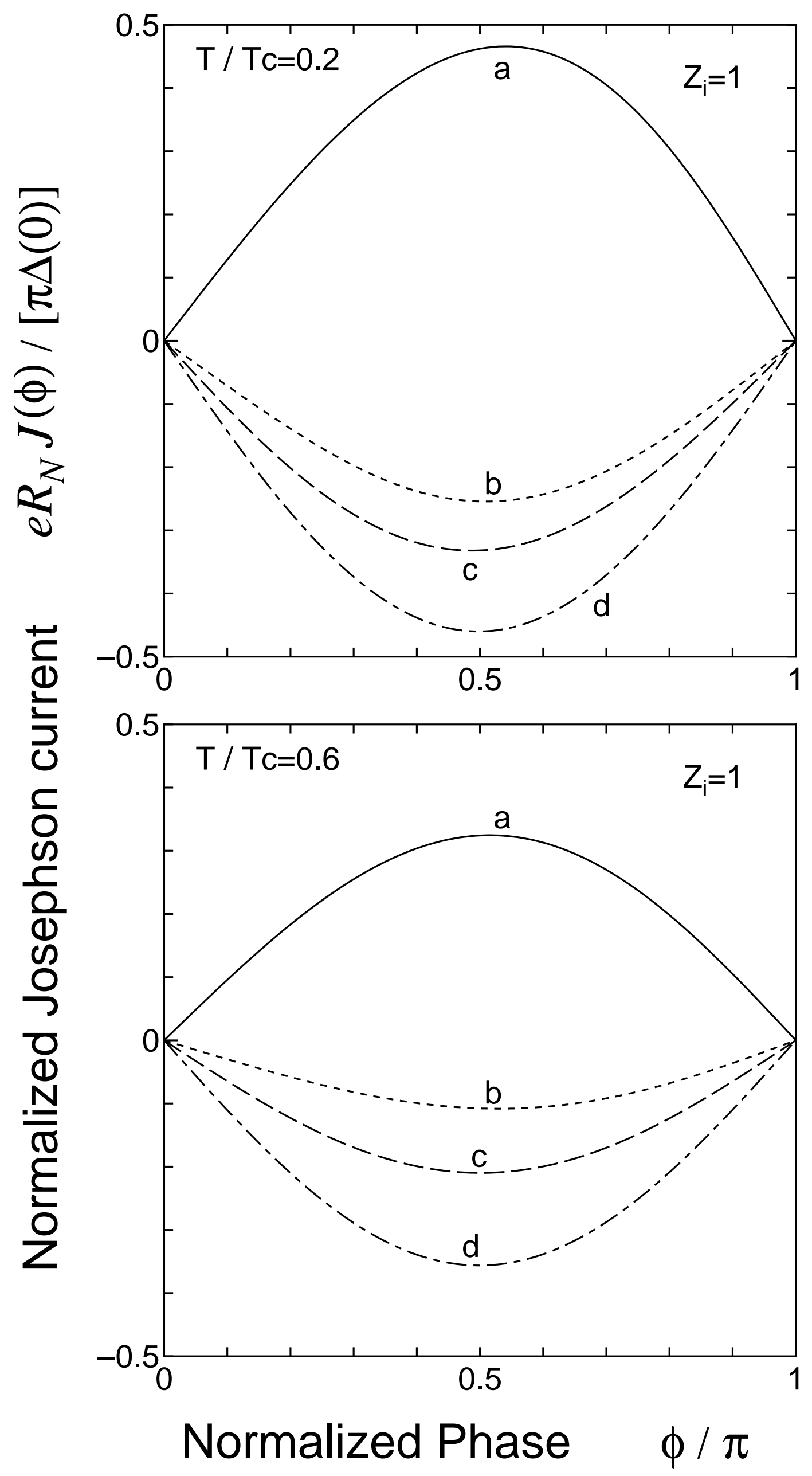


Normalized maximum Josephson current $e R_{N} J_{C} /[\pi \Delta(0)]$

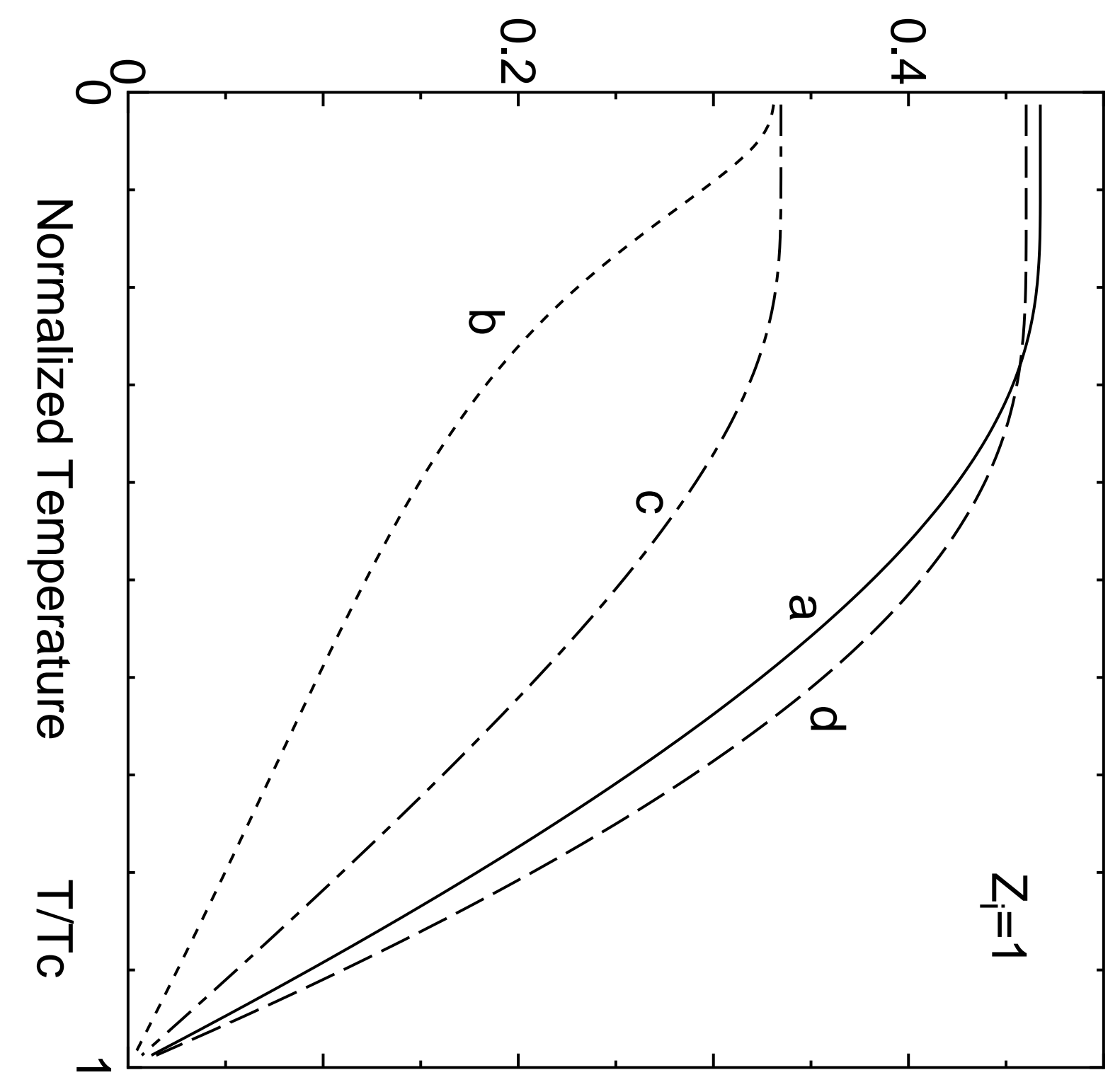


Normalized maximum Josephson current $e R_{N} J_{C} /[\pi \Delta(0)]$

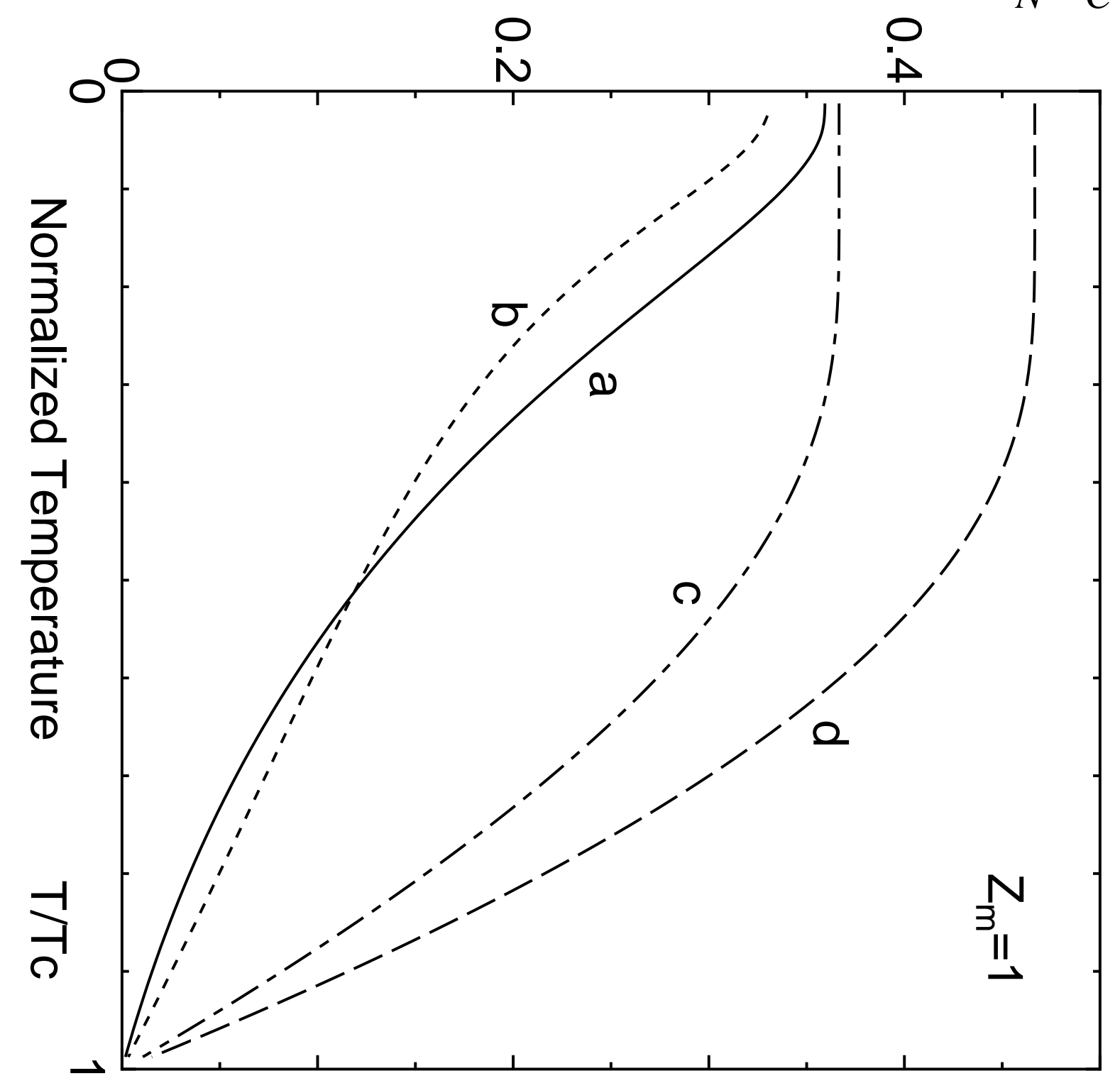

